\title{
Implementation of reconstructed radiance of IASI observations in WRF data assimilation system
}

\author{
Yu Yi ${ }^{1,2}$, Zhang Weimin ${ }^{1}$, Ye Minhua ${ }^{2}$, Sun jing ${ }^{2}$, Zhang Yukuan ${ }^{2}$ \\ ${ }^{1}$ Computer Science, National University of Defense Technology, Changsha, China \\ ${ }^{2}$ NO.94865 Troops of Chinese People's Liberation Army, Hangzhou, China \\ yuyi2019@nudt.edu.cn wmzhang104@163.com xyz01326@163.com 1073679954@qq.com \\ 550837288@qq.com
}

Keywords: Reconstructed radiances; IASI; Principal component; Data assimilation

\begin{abstract}
Methodology is explored for the reconstructed radiances from the Infrared Atmosphere Sounding (IASI) for the purpose of assimilation into numerical weather prediction models. A principal component based fast radiative transfer model has been implemented into the WRF data assimilation system, thus allow the investigation of the reconstructed radiances from the IASI observations. Testing of a prototype system with 165 reconstructed channel radiances in band 1 shows marginal improvement as there are a few channel-correlated errors existed in the reconstructed radiances even the noise reduced much. The method implemented in this regard is examined and the potential for channel selection for reconstructed radiances is explored.
\end{abstract}

\section{Introduction}

The Infrared Atmospheric Sounding (IASI) [1] is a kilochannel infrared Fourier transform spectrometer which was first launched on the MetOp-A satellite in October 2006. It measures the radiance emitted from the earth in 8461 channels covering the spectral interval from 645 to 2760 $\mathrm{cm}^{-1}(3.8-15.5 \mu \mathrm{m})$ at a resolution of $0.5 \mathrm{~cm}^{-1}$, providing information on atmospheric temperature, weather vapour content, ozone and trace gas concentrations with high accuracy and vertical resolution. IASI is the first truly operational Infrared (IR) interferometer on a weather satellite and is showed that it provides $25 \%$ of the impact of all observations, more than any other platform or conventional observation type [2].

Although IASI has become a major part of the assimilation system of many numerical weather prediction (NWP) centers [3][4], it might still be under-used because only a few hundred channels are carefully selected for data assimilation by prohibitive computational cost. These limited channels are far less than the full IASI spectrum, and current operational assimilation schemes for IASI are conservative.

In principle, the large number of channels from IASI with high spectral resolution and low radiometric noise mean that much more information about the atmospheric is available to improve the information content of the IASI assimilation. Reconstructed radiance [5] from the raw spectrum is proposed to represent the IASI observations more efficiently. The method has received a great deal of attention as a mean for assimilation of high-resolution infrared data. Here we design the structure for application the reconstructed radiance from IASI observations in the data assimilation system of Weather Research and Forecasting Model (WRFDA) [6] and test the prototype system.

The article is organized as follows: Sections 2 and 3 we review the theory of reconstructed radiance and discuss the features of reconstructed radiance derived from the raw IASI spectrum. Section 4 presents the methodology of assimilation of reconstructed radiance using the basic WRF data assimilation system. Finally, conclusions are given in section 5.

\section{Basic theory of reconstructed radiance}

Reconstructed radiances are calculated from the principal component (PC) scores [7]. Firstly eigenvectors are taken from a covariance matrix of radiance dataset, and then PC scores for each 
observation are computed for only the leading eigenvectors. The eigenvectors that are discarded mostly contain random measurement noise, thus the full spectrum of several thousand channels can be compressed into just a few hundred PC scores. Thirdly, the reconstructed radiances refer to truncated PC scores representation of the spectrum that has been converted back into radiance. These radiances are similar to the raw spectrum, but the truncation reduces the random noise in the measurements.

Suppose our dataset consists of 1 spectra of $n$ radiances arranged into an 1 by $n$ data matrix $R$. The dataset can then be represented by the vector population $r=(r 1, r 2, \ldots, r n)^{T} \quad($ here $T$ denotes the transpose). If $\mathrm{C}$ is $\mathrm{n}$ by $\mathrm{n}$ covariance matrix arranged as row vectors in descending order according to the magnitude of their eigenvalues, thus:

$$
C=\frac{1}{n_{\text {obs }}} R R^{T}=L \Lambda L^{T}
$$

Here, $\Lambda$ is the diagonal matrix of eigenvalues. $\mathrm{L}$ is the $\mathrm{PC}$ vector basis formed by the eigenvectors of the covariance matrix arranged as row vectors in descending order according to the magnitude of their eigenvalues.

The principal component, $\mathrm{P}$, related to the observations can be written as:

$$
P_{\mathrm{obs}}=L_{\mathrm{m}}^{T} R
$$

The PC vector basis $\mathrm{L}$ is then truncated and only chosen the first $\mathrm{m}(\mathrm{m} \leq \mathrm{n}$, in ranked order $)$ eigenvectors to compress the data and remove components (n-m) consisting mostly of random measurement noise. Typically, the radiance dataset would be the subset which consists of 165 long-wave channels in IASI band 1 covering the region between 645 and $875 \mathrm{~cm}^{-1}$ with a primary sensitivity to temperature and the surface. Each vector of observed PC scores has length $n=165$, but crucially we use only the highest rank PC scores $(1,2,3, \ldots, \mathrm{m})$ that convey most information about the atmospheric state to reconstruct the radiance $\mathrm{y}_{o b s}^{R R}$ of IASI observations:

$$
\mathrm{y}_{\text {obs }}^{R R}=L_{s} P_{\mathrm{obs}}=L_{\mathrm{s}} L_{\mathrm{m}}^{T} R
$$

\section{Assimilation methodology for reconstructed radiance}

\section{The architecture of data assimilation system for reconstructed radiance}

The WRF data assimilation seeks an "optimal" estimate of the true atmospheric state at the analysis time via the combination information of the observations over short time window and the corresponding model short-term forecast $\mathrm{Xb}$ on the model grid [8].

The atmospheric state $\left(X_{b}\right)$ is used as input to the fast radiative transfer model PCRT, and then to compute the model equivalent of the reconstructed radiance $Y_{\mathrm{xb}}^{R R}$. The observed IASI spectrum $\mathrm{R}$ is also inputted to the WRFDA system, and after the process of cloud screening and quality control the raw radiances are projected to the principal component space, and then the derived PC scores are transformed to reconstructed radiances $Y_{\mathrm{obs}}^{R R}$ from observations. If we ignore the time integration of the forecast model, the cost function to estimate the true atmospheric state through a process of minimization can be essentially:

$$
J(X)=\left[X-X_{\mathrm{b}}\right]^{T} B^{-1}\left[X-X_{\mathrm{b}}\right]+\left[Y_{\mathrm{obs}}^{R R}-Y_{\mathrm{xb}}^{R R}\right]^{T} O^{-1}\left[Y_{\mathrm{obs}}^{R R}-Y_{\mathrm{xb}}^{R R}\right]
$$

Here, $X_{b}$ is the background estimate of the atmospheric state and is described by the background error covariance $\mathrm{B}$. The error covariance $\mathrm{O}$ describes the accuracy of the observations. The atmospheric states Xa referred to as analysis value is derived from the minimization of the cost function. The departures of the observations $Y_{\mathrm{obs}}^{R R}$ from the simulated reconstructed radiance $Y_{\mathrm{xb}}^{R R}$ are referred to as observation innovation defined at the start of the 4D-Var window, and the simulated reconstructed radiances $Y_{\mathrm{xb}}^{R R}$ are derived from the observation operator $H_{R R}(X)$ by the following equation: 


$$
Y_{\mathrm{xb}}^{R R}=H_{R R}(X)
$$

The methodology adopted in this article for 4D-Var assimilation of reconstructed radiance is shown in Figure 1. The observed IASI radiances are first screened for the presence of clouds and the contaminated fields of view (FOVs) are discarded.



Fig. 1 The flow diagram of reconstructed radiance assimilation

\section{The observation operator PC_RTTOV model}

The observation operator applied in this paper is a PC-based fast radiative transfer model (PC_RTTOV) for the assimilation of Advanced TIROS Operational Vertical Sounder (ATOVS), and originally developed at the European Centre for Medium Range Weather Forecasts (ECMWF) in early 90's [9] for TOVS. The PC_RTTOV performs rapid and accurate simulation of PC scores and reconstructed radiances for hyper spectral infrared sounders using a multiple linear regression scheme. The assimilation system requires the use of a forward model referred to as observation operator $\mathrm{H}$ to simulate radiances, $\mathrm{H}(\mathrm{x})$, from the atmospheric state vector $\mathrm{x}$. The PC-based forward model $\mathrm{Hpc}(\mathrm{x})$ projects the radiances to $\mathrm{PC}$ space and the forward-modeled for reconstructed radiance $\operatorname{HRR}(\mathrm{x})$ project the $\mathrm{PC}$ scores to the radiance space by a matrix transform, thus:

$$
\begin{aligned}
& H_{P C}(x)=L_{m}^{T} H(x) \\
& H_{R R}(x)=L_{s} L_{m}^{T} H(x)
\end{aligned}
$$

Here, $Y_{\mathrm{xb}}$ is the radiance simulated by observation operator $\mathrm{H}$ from the atmospheric background profiles $\mathrm{X}$ on the observation grids. The simulation of the forward-modeled for reconstructed radiance includes the steps as follows:

- Input the atmospheric profiles to the standard fast radiative transmittance (RT) model.

- The standard fast RT model computes the polychromatic radiance in a multiple linear regression scheme.

- Calculate the PC scores by project the polychromatic radiance on the eigenvectors-based coefficients PC scores obtained from the covariance matrix of the large metrological dataset.

- Input the channel predictors, and transform the PC scores to be reconstructed radiance by also using the eigenvector-based coefficients.

The key step 4) is based on the equation (7) and the function is implemented as figure2. 


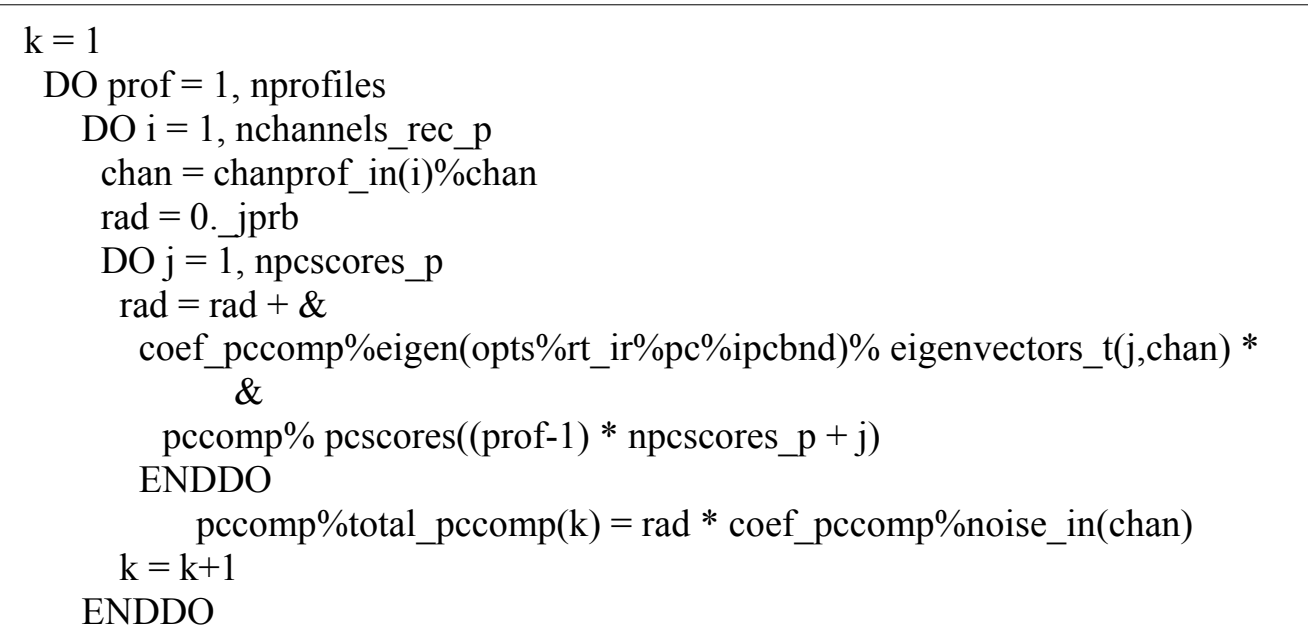

Fig.2 The algorithm based on reconstructed radiance.

\section{The error of reconstructed radiance}

If the raw observation vector has an error $\varepsilon_{y}$, the corresponding error for the reconstructed radiance is given by

$$
\varepsilon_{y}^{R R}=L_{s} L_{m}^{T} \varepsilon_{y}+\varepsilon_{R}
$$

Here, $\varepsilon_{R}$ is the error comes from the true atmospheric signal that is contained in the unused components. If the forward model that is used explicitly calculates reconstructed radiances, $\varepsilon_{R}$ is zero, although forward model error remains. If we carefully choose the number of principal components and the training set used to produce them, $\varepsilon_{R}$ should below the level of the first term in the equation and non-zero, we can assume that the reconstructed radiances are a 'noise-filtered' proxy for the raw radiances.

Reconstructed radiances have a different instrument error covariance from raw radiances, as the PC compression process introduces significant levels of correlation between channels and imposes mathematical constraints on the respective error covariance matrix $\mathrm{R}$, therefore

$$
\operatorname{Cov}\left(y^{R R}\right)=E\left[\varepsilon_{y}^{R R}\left(\varepsilon_{y}^{R R}\right)^{T}\right]=L_{s} L_{m}^{T} E L_{m} L_{s}^{T}+L_{s} L_{m}^{T} F L_{m} L_{s}^{T}
$$

Where $\mathrm{E}$ is the original observational error covariance matrix and $\mathrm{F}$ is the error covariance matrix introduced on reconstructing process. If the radiance are normalized by the true noise, then $\mathrm{E}=\mathrm{I}$. Despite these mathematic considerations, we used the IASI reconstructed radiances described in Collard et al. [6] in our assimilation experiments. We choose 165 discontinuous channels from channel 16 to channel 921 in band 1.

\section{Conclusions}

Testing of the reconstructed radiance based prototype system shows the noise level of the reconstructed radiances reduce significantly even only marginal improvement in forecast skill above the use of the raw radiance. That would be the reason that reconstructed radiance would tend to have channel-correlated errors and the Collard method of channel selection was generated for raw radiances, where there are no constraints on the rank of the error covariance matrix. In the future work we will research more methods for channel selection based on mathematical and physical consideration. 


\section{Acknowledgements}

This study was supported by the Special Scientific Research Project for Public Interest (GYHY201006015), the National Natural Science Foundation of China (Grant Nos.41305101).

\section{References}

[1] G. Chalon, F. Cayla, D. Diebel, 'IASI': An advanced sounder for operational meteorology. I n Proc. of the 52nd Congress of IAF, Toulouse, France, 1-5. http: // smsc . cnes.fr / IASI / A publications . htm, October 2001.

[2] S. Joo, J. Eyre, and R. Marriott, The impact of Metop and other satellite data within the Met office global NWP system using an adjoint-based sensitivity method, Mon. Wea. Rev., Vol.144, pp. 3331-3342, DOI: http: //dx.doi.org/:10.1775/MWR-D-00232.1, 2013.

[3] AD Collard, AP McNally, The assimilation of Infrared Atmospheric Sounding Interferometer radiance at ECMWF, Q. J. R. Meteorol. Soc. 135:1044-1058, 2009.

[4] FI Hilton, NC Atkinson, SJ English, JR Eyre, Assimilation of IASI at Met Office and assessment of its impact through observing system experiments, Q. j. R. Meteorol. Soc. 135:495-505, 2009a.

[5] AD Collard, AP McNally, FI Hilton, SB Healy, NC Atkinson, The use of principal component analysis for the assimilation of high-resolution infrared sounder observations for numerical weather prediction, Q. j. R. Meteorol. Soc. 136:2038-2050, 2010.

[6] XY Huang, Q. Xiao, DM Barker, X Zhang, J Michalakesd, W Huang, T Henderson, J Bray, Y Chen, Z Ma, J Dudhia, Y Guo, X Zhang, DJ Won, HC Lin, YH Kuo, Four-Dimension Variational Data Assimilation for WRF: Formulation and Preliminary Results, Mon. Wea. Rev. 137. 299-314, 2009.

[7] G. Masiello, C. Serio, and P. Antonelli, Inversion for atmospheric thermody-namical parameters of IASI data in the principal components space, Q. j. R. Meteorol. Soc., Vol. 138, pp. 103-117, DOI: http://dx.doi.org/10.1002/qj.909, 2012.

[8] Y Yi, ZH Weimin, W Zhongyuan, Y Xiaofeng, C Xiaoqun, ZH Mengbin, Assimilation of HY-2A scatterometer sea surface wind data in a 3DVAR data assimilation system-A case study of Typhoon Bolaven, Front. Earth Sci. DOI: 10.1007/s11707-014-0461-8, 2014.

[9] JR Eyre, A fast radiative transfer model for satellite sounding systems, ECMWF Technical Memorandum 176, 1991. 